\title{
Serum antibodies to Legionella agents in bronchial asthma
}

\author{
S BEER, I BOLDUR, R KAZAK, S AVIDAN, AND Y KANNAI
}

Paediatric Pulmonary-Allergic Service, Department of Microbiology, Assaf Harofeh Medical Center, Sackler School of Medicine, Tel Aviv; and Department of Theoretical Mathematics, Weizman Institute of Science, Rehovot, Israel

SUMmARY The prevalence of seropositivity to Legionella species was studied in 184 children with bronchial asthma and 80 control children, age and sex matched, without respiratory tract infections. The sera were examined by indirect immunofluorescence with antigens of six Legionella species. The asthmatic children showed a significantly high percentage of seroreactions to $L$ pneumophila compared with the control children. In the asthmatic children, no association was found between age, sex, onset of asthmatic symptoms, living conditions, corticosteroid treatment, or exposure to aerosols and seropositivity to Legionella. No relation could be found between the asthma severity score and the titre of serum antibodies to L pneumophila.

None of the 32 sera with titres of 1:256 or higher were reactive for Legionella specific IgE antibodies.

A high prevalence of antibodies to $L$ pneumophila was found in children suffering bronchial asthma. Further studies are necessary to clarify the clinical relevance.

Since the aetiological agent of legionnaires' disease was first described in adult patients in $1975,{ }^{1}$ a few cases have been reported in childhood. ${ }^{2-4}$ In a previous survey from our hospital, three of 167 children admitted to hospital for acute respiratory infections, had antibody titres of 1:256 or more to Legionella species at the time of admission; one of these children had asthma. ${ }^{5}$ Respiratory tract infections have a particularly important impact upon children with asthma. Recently antibody titres of $1: 256$ or higher to $L$ pneumophila were reported in $29.4 \%$ of children with cystic fibrosis. ${ }^{6}$ A study of 113 adults suffering from chronic pulmonary diseases, including 41 with bronchial asthma, showed a significant increase of serum antibodies to $L$ pneumophila. $^{7}$

This study was designed to determine whether asthmatic children show a higher incidence of seropositivity to Legionella species compared with non-asthmatic children. Precipitating asthma attacks in children are often associated with symptoms that may be clinically regarded as evidence of upper or lower respiratory infections, but while the role of viral infections in precipitating asthma attacks is already well established, the role of bacterial infections is not yet clear.
As far as we know, this is the first prevalence study of antibodies to Legionella species in children with bronchial asthma.

\section{Patients and methods}

Study population. Serum samples were collected during one year (August 1982 to August 1983) from 184 asthmatic children attending the outpatient clinic of the Paediatric Pulmonary-Allergic Service regularly.

The sera were obtained when blood was drawn for other diagnostic examinations. All children were suffering from bronchial asthma according to the criteria of the American Thoracic Society. ${ }^{8}$ A total of 134 children had perennial extrinsic allergic asthma and 50 had seasonal allergic extrinsic bronchial asthma. All the specimens were collected when the children were free of symptoms. Of the 184 children, $118(64 \cdot 14 \%)$ were boys and $66(35 \cdot 86 \%)$ were girls; their ages ranged from 2 to 15 years (mean 8.3 years). The first asthmatic attack had occurred in $56.5 \%$ of the patients three or more years before the examination, and in $29.1 \%$ of the patients one to two years earlier. The parents were asked to answer a standardised questionnaire on the 
severity of asthma during the preceding year. An asthma severity score from 1 to 14 was computed for each child, as described by Murray et al.$^{9}$ Additional information gathered included the number of past admissions to hospital, previous use of mist tents or drug administration by aerosols, and mean age from onset of symptoms. Although corticosteroid treatment is one of the asthma severity score's features, it was considered separately also, because of its particular importance in legionnaires' disease.

Eighty children suffering from various nonrespiratory disorders, or surgical patients, age and sex matched with the study group, served as controls. The study was possible because blood was drawn for other routine examinations; however, informed consent was obtained from the parents of both the study and the control group.

Laboratory tests. The serum samples were stored at $-20^{\circ} \mathrm{C}$ until examined by the indirect immunofluorescence assay. Antigens and positive antisera for standardisation were obtained from the Centers for Disease Control, USA, and represented $L$ pneumophila, gr 1-4 (monovalent and pool); $L$ pneumophila, gr 5-6 (monovalent and pool); $L$ longbeachae, gr 1-2 (pool); and L micdadei, $L$ gormanii, $L$ dumoffii, and $L$ bozemanii. The immunofluorescent assay was examined according to the technique of the Centers for Disease Control (1981), with the exception that the first screening was performed with a commercial conjugate (Institute Pasteur, Paris) fluorescein isothiocyanate antihuman gammaglobulin ( $\mathrm{Ig})$, and those positive to a titre of 1:256 or more were examined again after absorption with antigen of Escherichia coli 013:k92: $\mathrm{H} 4{ }^{10}$ This procedure excluded eight sera $(19 \cdot 5 \%)$ from the group positive to a titre of 1:256 or more, as their titres after absorption were 1:128. Sera positive to $1: 256$ or more with fluorescent rabbit anti-Ig were examined further for IgG- and IgMtype antibodies with fluorescein isothiocyanate antihuman IgG (antigamma) and antihuman IgM (anti$\mu \mathrm{u})$ (Wellcome Reagents). The two sera positive for IgM were absorbed on a column of polymerised human $\mathrm{IgG}^{11}$ to avoid false reactions due to rheumatoid factors. Only one of them was refractory to this treatment and is considered positive to IgM. The positive sera with titres of 1:256 or higher were also examined for antibodies to Chlamydia, Мycoplasma, and Pseudomonas aeruginosa serogroup H11 to rule out false positive reactions. The complement fixation test for Chlamydia and Mycoplasma was performed by the Public Health Laboratories at Abu Kabir. Pseudomonas antibodies were tested by immunofluorescent assay with goat antihuman IgG ( $\gamma$ chain specific) fluorescein isothiocyanate conju- gate, affinity chromatography purified (Sigma Chemical, USA). One child had low titres for Chlamydia (1:120) and Mycoplasma (1:60). Three children had a titre of 1:64 for $P$ aeruginosa; these results probably did not interfere with the titres to Legionella.

All the sera with titres of 1:256 or more were tested for IgE specific antibodies to L pneumophila. We used the immunofluorescent assay first, performed as described for IgG and IgM. Goat antihuman $\operatorname{IgE}$ ( $\varepsilon$ chain specific) fluorescein isothiocyanate conjugate was from Miles Yeda, Israel. ${ }^{12}$ This antiserum was absorbed before use with a mixture of formalin-killed $L$ pneumophila cells, serogroup $1-4$, because it cross reacted with $L$ pneumophila.

The tests for specific IgE for $L$ pneumophila were performed by two enzyme linked immunoassays (ELISA) because we were not in possession of a positive control. The first system was as described by Farshy. ${ }^{13}$ The plates used were from Dynatech (M/129/B). They were coated with soluble $L$ pneumophila serogroup 1-4 antigen. ${ }^{14}$ Horse radish peroxidase conjugated to goat antihuman IgE (heavy chain specific) was from Miles Yeda, Israel. The results were read spectrophotometrically at 492 $\mathrm{nm}$ with a Gilford (2600) spectrophotometer. The second ELISA system used was part of the Platelia total IgE kit for the quantitative determination of total IgE in human serum (Institute Pasteur, Paris). The kit was used as recommended by the manufacturer, in addition to the coated plate method, described above.

\section{Results}

The seroreactions of the study groups to $L$ pneumophila are summarised in Table 1. Significantly more asthmatic children were seroreactive to Legionella antigens. The $\chi^{2}$ test was used for statistical analysis. Thirty two $(17.4 \%)$ asthmatic children and only one $(1.25 \%)$ of the children from the control group had titres of 1:256 or more $(P<0 \cdot 001)$. Four children of the 32 had titres of $1: 512$. One of them reacted with IgM at $1: 256$. Thirty seven $(20 \cdot 11 \%)$ of the asthmatic children had a titre of $1: 128$ compared with four $(5 \%)$ in the control group $(\mathrm{P}<0.01)$. Eighty five $(46 \cdot 1 \%)$ of the asthmatic children had titres of 1:64 compared with 15 children $(18.75 \%)$ in the control group $(\mathrm{P}<0.005)$. Thirty $(16.3 \%)$ of the asthmatic and 60 $(75 \%)$ of the control group children, had titres of less than 1:64.

The differences between the frequency of given titres of the asthmatic children versus the healthy children are shown in Fig. 1. The cumulative 
Table 1 Seroreactions to Legionella pneumophila

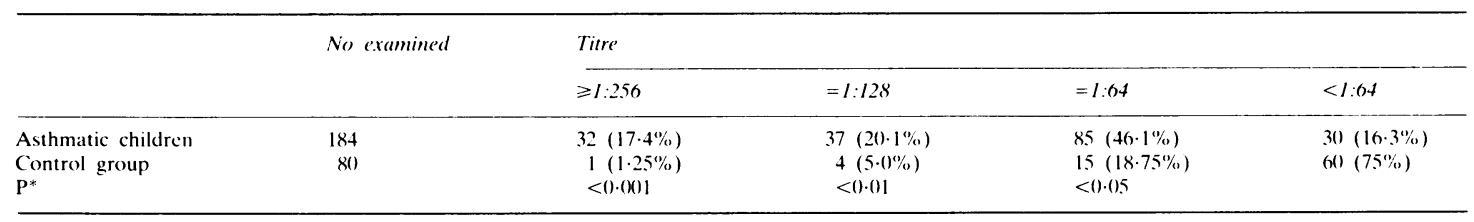

$\mathrm{P}^{*}$ calculated by $\chi^{2}$ test.

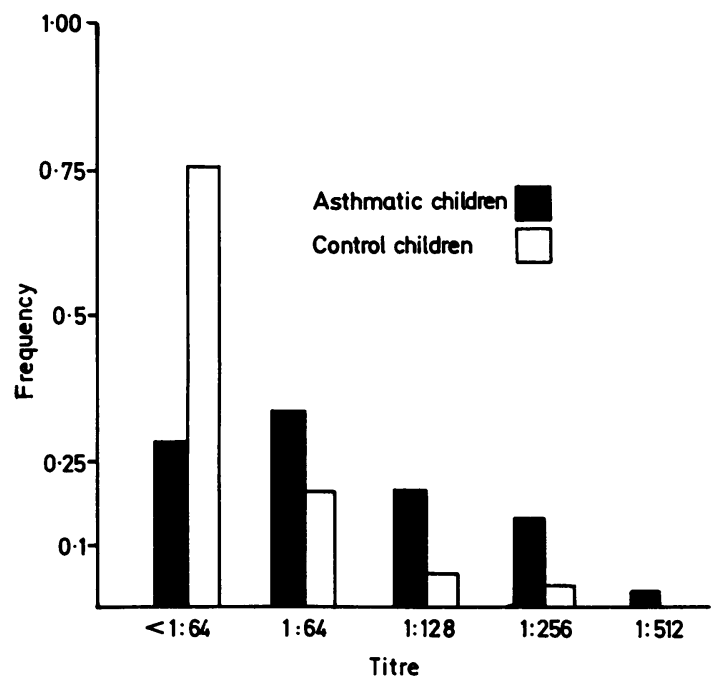

Fig. 1 Frequencies of given titres for the asthmatic and the control children.

distributions are shown in Fig. 2. The difference between the two groups calculated by Smirnov's test is highly significant $(\mathrm{P}<0 \cdot 000001)$.

Of the 32 positive reactions to $L$ pneumophila (titre 1:256 or more) listed in Table 1 two sera were monovalent (serogroup 1); two reacted equally with serogroups 3 and 4 ; two reacted equally with serogroups 5 and 6 ; and one reacted equally with serogroups 1 and 5. Four sera reacted with three serogroups: one of them equally high for serogroups 2 and 4 and less for serogroup 5; two equally high for serogroups 3 and 4 and less for serogroup 6; and one equally high for serogroups 5 and 6 and less for serogroup 2. Nine sera reacted with all monovalent antigens of serogroup 1-4 and six reacted with all serogroups $1-6$ with different titres.

Two asthmatic children had titres of $1: 64$ to $L$ micdadei, one to $L$ bozemanni, and one to $L$ longbeachae. One asthmatic child had a titre of 1:128 for $L$ bozemanii. Three children from the control group had titres of 1:64 for $L$ bozemanii and one had a titre of 1:128 for the same agent.

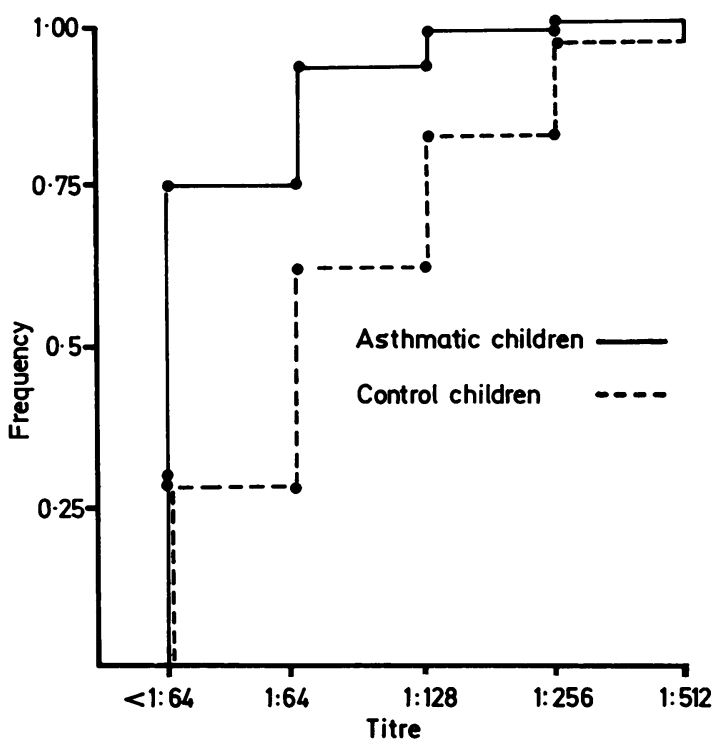

Fig. 2 Cumulative distributions of the frequencies of given titres for the asthmatic and the control children.

In the asthmatic children, the serological titres were unrelated to age and sex. The mean age and mean age in years from the onset of symptoms are shown in Table 2. The percentage of children with perennial asthma was more or less constant in each titre group. No association was found between the mean number of years from the onset of the first asthmatic symptoms and the titre group (Table 2).

Using the asthma history scores versus the titre of serum antibodies to $L$ pneumophila, a regression line, based on all the observations, was computed with the Statistical Analysis System (SAS) general model's procedure (Fig. 3). The significance level of the intercept is 0.0001 and the slope 0.0003 . The correlation coefficient $\mathrm{r}=\mathbf{0} \cdot 265$.

The 32 sera with titres of 1:256 or more were tested further for IgE specific anti-Legionella antibodies. None of those sera was found reactive by any of the three methods performed. No difference was found between patients with high or low concentrations of $\mathrm{IgE}$. 
Table 2 Age and onset of symptoms related to seroreactivity to Legionella pneumophila in asthmatic children

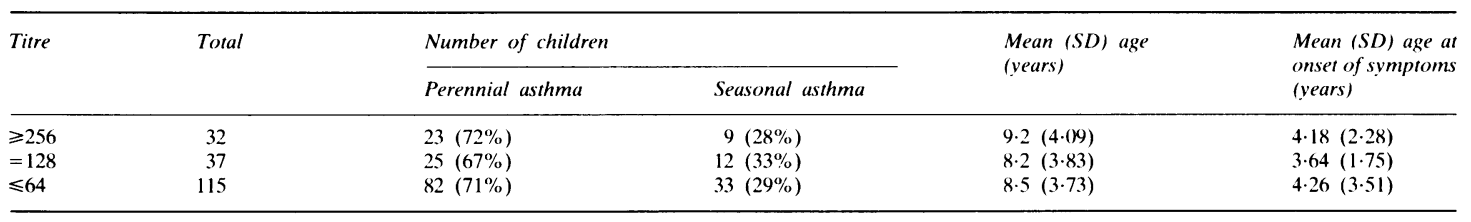

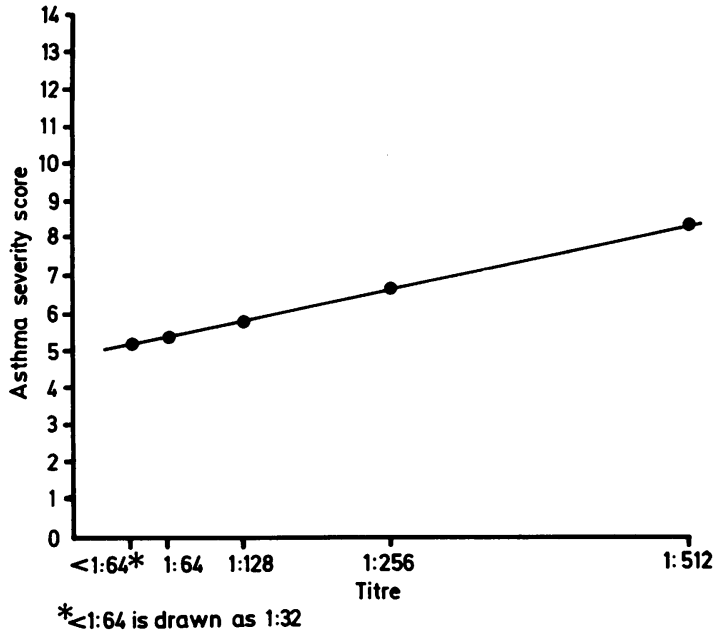

Fig. 3 Regression line of asthma severity score verus titre of serum antibodies to Legionella pneumophila

\section{Discussion}

A serological titre of $1: 256$ or higher is accepted by the Centers for Disease Control as presumptive for a present or past infection with Legionella. ${ }^{15}$ Half of the survivors of the Philadelphia outbreak, however, had a titre of less than $1: 128$ after two years. ${ }^{16}$ Therefore presumptive titres for past infection are arbitrary and have to be established according to the background titres found in a given population.

This area is not regarded as endemic for legionnaires' disease, and prevalence of seroreactions to Legionella antigens is presumably due to nosocomial infections in subjects with long term institutional contact. ${ }^{17} 18$ For all these reasons we did not establish cut off limits for seropositivity, but we compared the percentage of asthmatic children seroreacting with a particular titre with the percentage of healthy children seroreacting with the same titre.

The asthmatic children show a very high incidence of seroreactivity to Legionella species compared with children free of respiratory diseases (Table 1, Figs. 1 and 2) and with children with acute respiratory infections, ${ }^{5}$ from the same hospital.
Our results are highly significant calculated by either $\chi^{2}$ test (Table 1) or Smirnov test, which detects discrepancies between two empirical distribution functions. Seropositivity to Legionella belonging to groups other than $L$ pneumophila was very low in our study groups, therefore we analysed statistically only the seroreactions to this.

Previous studies have shown increases in antibody titres during convalescence from legionellosis that were specific for a single serogroup antigen or against antigens common in multiple serogroup strains. ${ }^{15} 19$ The seropositive sera in our study cross reacted with the individual antigens belonging to $L$ pneumophila with relatively high frequency; as in the other prevalence studies performed in our area. ${ }^{17} 18$ It has been proposed, but not proved, that the common antigen reactions may be secondary reactions on repeat exposure to a different serogroup strain. ${ }^{19}$

An analysis of the accumulated data on the asthmatic group of children showed no relation between the mean age or between the mean number of years from the onset of the first asthmatic symptoms and the titre group (Table 2).

The correlation coefficient obtained between the asthma severity score and the antibody titre to $L$ pneumophila from the regression line in Fig. 3. is small $(\mathrm{r}=0 \cdot 265)$, that means that there is a very high variability in the data.

Legionnaires' disease pneumonia is acquired mainly by inhalation of aerosols, from waterborne environmental sources, as condensate from air cooling systems, or central air conditioning systems. None of the asthmatic children lived in such conditions. The association found between serological titres and number of past admissions to hospital, exposure to aerosols in the emergency room, or number of lower respiratory tract infections among the asthmatic children is poor.

Corticosteroid treatment, one of the score's features, ${ }^{9}$ is considered to be one of the predisposing factors in legionnaires' disease, but no relation was found between corticosteroid treatment alone and the titre group of the asthmatic children. In a study of 41 adult patients with asthma, $20 \%$ had raised antibody titres against $L$ pneumophila. This prevalence of titres of 1:64 or more among patients 
with asthma was not associated with corticosteroid treatment. ${ }^{7}$ In another study of young adults with cystic fibrosis, no relation was found between the presence of raised antibody titres to Legionella and corticosteroid treatment. ${ }^{20}$

We have no evidence that the higher seroreactivity of the asthmatic children is due to their higher susceptibility to infection with Legionella. An explanation for the high incidence of seropositivity to Legionella in asthmatic children, could be overactivity of the immune system reflected by raised immunoglobulin concentrations, especially IgE. Using three different techniques, no serum IgE antiLegionella antibodies were detected in the 32 sera with titres equal to or higher than 1:256. No correlation was found between the antibody titre to Legionella and the concentration of total serum IgE of the asthmatic children.

We could not obtain any reference to this subject through the computerised Allergy Service Pharmacia Information Program. The incidence of $\mathrm{IgE}$ antibodies in bacterial disease is not well documented. Some authors claim that bacterial infections play little role in precipitating asthma attack. ${ }^{21}$ Others found IgE antibodies to Haemophilus influenzae and Streptococcus pneumonia, and therefore conclude that immediate hypersensitivity to bacteria may play a role in the infectious exacerbations of bronchial asthma. ${ }^{22}$ Some authors suggest that IgE antibacterial antibodies appear early in the course of infectious disease, somewhat later than IgM but earlier than IgG antibodies. ${ }^{12}$ This could be the reason why we did not find positive sera for IgE specific to Legionella. The sera were collected from the asthmatic patients in intervals between the asthmatic attacks and not immediately after a mild or acute respiratory disease.

One case of legionnaires' disease associated with severe bronchospasm and hypoventilation was reported in an adult patient. ${ }^{23}$ Further studies are necessary to prove the role of Legionella in infections during exacerbations of asthma.

It should, however, be kept in mind that asthma patients have long term contact with the infected hospital environment, either as outpatients or as inpatients; they are often treated with corticosteroids which have an immunosuppressive character and are frequently inhaling medications through aerosols which are recognised sources for legionnaires' disease. All these together lead to a continuous exposure of asthmatic children to Legionella and could be a contributing factor for their higher incidence in seropositivity than healthy children.

Many febrile respiratory diseases in asthmatic children remain without a differential diagnosis.
The high prevalence of positive seroreactions to Legionella antigens described in our study indicates that Legionella infections should be considered in diagnosis and treatment.

We thank the chief nurses $L$ Cohen, E Bonda, and $Y$ Baruch for their kind cooperation, and Mrs J Szichman for the secretarial help.

\section{References}

1 Fraser DW, Tsai TR, Orenstein W, et al. Legionnaires' disease. Description of an epidemic pneumonia. N Engl J Med 1977;297: 1189-97.

2 Ryan ME, Feldman S, Pruitt B, Fraser DW. Legionnaires' disease in a child with cancer. Pediatrics 1979;64:951-3.

${ }^{3}$ Simpson RM, Cogswell JJ, Mitchell ER, Macrae AD. Legionnaires' disease in an infant. Lancet 1980;ii:740-1.

${ }^{4}$ Muldoon RL, Jaecker DL, Keifer HK. Legionnaires' disease in children. Pediatrics 1981;67:329-32.

5 Mundel G, Goldberg A, Boldur I, Sompolinsky D, Katz Y. Legionnaires' disease in Israel: serological evidence of childhood infection. Isr J Med Sci 1983;19:380-2.

${ }^{6}$ Katz SM, Holsdaw DS. Serum antibodies to Legionella pneumophila in patients with cystic fibrosis. JAMA 1982;284: 2284-8.

7 Freedman AP, Katz SM. The prevalence of serum antibodies to Legionella pneumophila in patients with chronic pulmonary disease. Am Rev Respir Dis 1981;123:238-9.

8 American Thoracic Society. Definition and classification of chronic bronchitis, asthma and pulmonary emphysema. Am Rev Respir Dis 1962;85:762.

${ }^{9}$ Murray AB, Fergusson AC, Morisson B. Airway responsiveness to histamine as a test for overall severity of asthma in children. J Allergy Clin Immunol 1981;68:119-24.

${ }^{10}$ Wilkinson HW, Farshy CE, Fikes BJ, Cruce DD, Yealy LP. Measure of immunoglobulin G-, M- and A- specific titers against Legionella pneumophila and inhibition of titers against nonspecific gram negative bacterial antigens in the indirect immunofluorescence test for legionellosis. J Clin Microbiol 1979;10:685-9.

"Merlini G, Forsgren A, Turesson I, Zettervall O. An IgM monoclonal protein with multiple serological specificities. Clin Exp Immunol 1979;37:276-82.

12 Escende A, Serre A. IgE anti-brucella antibodies in the course of human brucellosis and after specific vaccination. Int Arch Allergy Appl Immunol 1982;68:172-5.

${ }^{13}$ Farshy CE, Feeley JC. ELISA for Legionnaires' disease antibody and antigen detection systems: an interim report. In: GL Jones, GA Hebert, eds. 'Legionnaires': the disease, the bacterium and methodology. Atlanta: Public Health Service, (US Department of Health), 1979:123-9.

${ }^{14}$ Farshy CE, Klein GC, Feeley JC. Detection of antibodies to the Legionnaires' disease organism by microagglutination and micro-ELISA tests. J Clin Microbiol 1978;7:327-31.

15 Wilkinson HW, Fikes BJ, Cruce DD. Indirect immunofluorescence test for serodiagnosis of Legionnaires' disease: evidence for serogroup diversity of Legionnaires' disease bacterial antigen and for multiple specificity of human antibodies. J Clin Microbiol 1979:9:379-83.

${ }^{16}$ Lattimer GL, Rhodes LV, Salventi JS, et al. The Philadelphia epidemic of Legionnaires' disease: clinical pulmonary and serological findings two years later. Ann Intern Med 1979;90: 522-6.

17 Boldur I, Modai D, Iaine A, et al. Legionnaires' disease in Israel: a serological study of hemodialysis patients. Isr J Med Sci 1982;18:667-70.

${ }^{18}$ Boldur I, Ergaz M, Sompolinsky D. A prevalence study of antibodies to Legionella in geriatric institutions. J Hyg Camb 1984;92:37-43. 
19 McKinney RM, Wilkinson HW, Sommers HM, et al. Legionella pneumophila serogroup six: isolation from cases of legionellosis, identification by immunofluorescence staining, and immunological response to infection. J Clin Microbiol 1980;12:385-401.

20) Efthimiou J, Hodson EM, Taylor P. Taylor AG, Batten JC. Importance of viruses and Legionella pneumophila in respiratory exacerbations of young adults with cystic fibrosis. Thorax 1984;39:150-4.

21 Welliver CR. Upper respiratory infections in asthma. $J$ Allergy Clin Immunol 1983;72:341-6.
22 Pauwels R, Verschraegen G, Van Der Straeten M. IgE antibodies to bacteria in patients with bronchial asthma. Allergy 1980;157:665-9.

23 Rosenberg MD, Martin L, Lerner Ph, Parker MD. Legionnaires' disease. Association with severe bronchospasm and hypoventilation. Chest 1982:82:383.

Correspondence to Dr S Beer, Paediatric Pulmonary-Allergic Service, Assaf Harofeh Medical Center, Zerifin 70300, Israel.

Received 14 November 1984

(⿸丆口⺕亅 